This document is published in:

Fusion Engineering and Design (2010) 85 (7-9) 1659-1663.

DOI: 10.1016/j.fusengdes.2010.05.010

(C) 2010 Elsevier B.V. 


\title{
TechnoFusión, a relevant facility for fusion technologies: The remote handling
} area

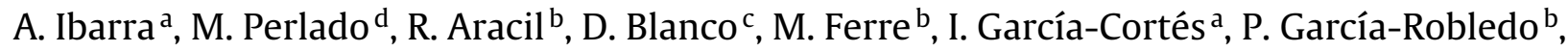 \\ M. González ${ }^{\text {a,* }}$, P. González ${ }^{\mathrm{e}}$, D. Jiménez-Rey a , J.L. Martinez-Albertos ${ }^{\mathrm{a}}$, L. Moreno ${ }^{\mathrm{c}}$, \\ J. de No ${ }^{\mathrm{e}}$, V.M. Queral ${ }^{\mathrm{a}}$, L. Ríos ${ }^{\mathrm{a}}$, R. Román ${ }^{\mathrm{a}}$ \\ a LNF-CIEMAT, Av. Complutense 22, 28040 Madrid, Spain \\ b DAIEII, ETSII, UPM, C/José Gutiérrez Abascal 2, 28006 Madrid, Spain \\ ${ }^{c}$ LEMI-UC3M, Av. Universidad Carlos III, 22, 28270 Colmenarejo, Spain \\ d IFN, ETSII, UPM, C/José Gutiérrez Abascal 2, 28006 Madrid, Spain \\ e IAI-CSIC, Ctra. Campo Real, km 0,200, 28500 La Poveda, Spain
}

\begin{abstract}
The future commercial production of electricity based on thermonuclear fusion requires the development of a number of research projects in addition to ITER, mostly related to the development of long-term technologies needed for future fusion reactors. Among the priority areas identified in the framework of international fusion research programmes, it is projected to build a specific research centre for fusion technologies in Spain. The so-called National Centre for Fusion Technologies, TechnoFusión, will be equipped with a large number of top facilities for the fusion technological development. The research activities will be focused primarily on several areas, including the implementation of advanced man-ufacturing technologies, the evaluation of radiation effects on low activation structural and functional materials, the in-beam and out-beam characterization of materials, the development of robotics and automated systems for remote handling, studies of liquid metal technologies and computer simulation. Currently no similar facilities to TechnoFusión exist, so it will provide more realistic tests than those available in other facilities up today, helping in the fast track to DEMO and IFMIF.

The present document summarizes a review of the different facilities to be included in TechnoFusion, with special emphasis on those proposed for remote handling $(\mathrm{RH})$ applications. The paper will review the technical specifications concerning the RH facility, the analysis of the mock-up components and tests to be performed, and the relevance of the RH lab capabilities, particularly the required equipment under irradiation conditions.
\end{abstract}

Keywords: Fusion nuclear technologies, Remote handling

\section{Introduction}

A present approach to fusion power [1,2] is to simultaneously construct and operate ITER (the International Thermonuclear Experimental Reactor) and IFMIF (the Irradiation Fusion Materials Facility) in order to provide information for an early decision on DEMO (the DEMOnstration power plant), the device [3] that will bridge the gap between ITER and the first-of-a-kind fusion power plant (FPP). There are discrepancies on the objectives of DEMO, being one of the main objectives to qualify components and processes in reactor relevant conditions for the first FPP. As a result, in the next decades, the main fusion research efforts will be focused on the development of technological components for future reactors, rather than in basic plasma physics.
The reliability and availability of a FPP will depend on the behaviour of complex components, such as first wall materials or breeding blanket modules in the combined presence of thermal, electromagnetic and radiation-induced effects, as well as the remote handling systems working under extreme conditions. The behaviour of these components under the mentioned limiting factors is largely unknown and requires great Research and Development efforts. In an attempt to contribute on the priority areas identified in the framework of international fusion research programmes, it is projected to build a singular research and technological centre, The National Centre for Fusion Technologies, TechnoFusión, in Spain. Technofusión is proposed as a new relevant triple-beam ion irradiation and technological facility able to screen radiation damage phenomena relevant for IFMIF and DEMO environments. This paper summarizes a brief review of the different facilities to be included in TechnoFusión, with special emphasis on those proposed for remote handling (RH) applications. 


\section{TechnoFusión}

The Spanish Ministry of Science and Innovation, in collaboration with the regional government of Madrid, Spain, approved in January 2007 the construction of several facilities as essential tools for the competitive and qualitative development of science research and technology. TechnoFusión, one of these singular scientific and technological facilities, will create the infrastructure required to develop the technologies needed in future commercial fusion reactors, assuring the participation of Spanish research groups and companies. Currently no similar facilities to TechnoFusión exist, so it will provide more realistic tests than those available in other facilities up today, helping in the fast track to IFMIF and DEMO.

TechnoFusión consists of seven large experimentation areas, many of which are unique in the world, with the following main technical objectives.

\subsection{Material production and processing}

The uncertainty about the materials to be used in the future fusion reactors make it of utmost importance to implement installations capable of manufacturing new materials on a semiindustrial scale. Top priority materials to be produced are oxide dispersion strengthened (ODS) steels and tungsten alloys. This type of plant requires special equipment currently scarce in Spain: vacuum induction furnaces, hot isostatic pressing furnaces, pulsedplasma-current-assisted sintering furnaces, or vacuum plasma projection systems.

\subsection{Irradiation of materials}

The main purpose of this area is to simulate the radiation effects in a reactor by irradiation with ions and electrons and light ion implantation. Two tandem linear accelerators, with terminal voltages of $6 \mathrm{MV}$ to implant $\mathrm{He}, \mathrm{H}$ and $\mathrm{D}$, and a heavy ion accelerator ( $k=110,{ }^{1}$ cyclotron-type) to irradiate with heavy ions ( $\mathrm{Fe}, \mathrm{W}, \mathrm{Si}$, $C)$ and high energy protons will be the needed equipment. An additional high magnetic field, between 5 and $10 \mathrm{~T}$, must also be implemented to study the simultaneous effect of radiation and magnetic fields on materials. The effects of ionizing gamma radiation will be simulated by a Rhodotron ${ }^{\circledR}$ electron accelerator with a fixed energy of $10 \mathrm{MeV}$, to be shared with other areas of TechnoFusión.

\subsection{Plasma-wall interactions}

Inside a fusion reactor, some materials will not be subjected only to radiation, but also to enormous heat loads in the case of divertor materials and plasma disruptions. In view of this, both stationary conditions-due to its intrinsic properties: high density, low temperature and high power-and violent transient events (edge-localized modes or ELMs) must be reproduced. Therefore, two devices for $\mathrm{H}, \mathrm{D}$, He or Ar plasma generation are planned for TechnoFusión: one is a linear plasma device, meant to reproduce the above-mentioned stationary conditions, and the other is a quasi-stationary plasma accelerator (QSPA) to simulate the transients.

\footnotetext{
$1 k$, proton kinetic energy.
}

\subsection{Liquid metal technologies}

A number of components of ITER, DEMO and IFMIF will use liquid metals as refrigerants, tritium generators, neutron reproducers, moderators, etc., all of them under extreme conditions. Therefore, these applications need further research to be finally implemented. The main goals of this area are the studies of (i) the free surface of liquid metals under conditions of internal energy deposition and (ii) the compatibility of structural materials and liquid metals in the presence of radiation. It will also be possible to study the influence of magnetic fields on the mentioned phenomena as well as the development of methods for (i) purification of liquid metals, (ii) enrichment of lithium, (iii) extraction of tritium, and (iv) development of safety protocols for liquid metal handling. The basic working scheme for this area in TechnoFusión facility is an arrangement of several liquid lithium loops, one of them associated to the Rhodotron ${ }^{\circledR}$ electron accelerator, to investigate the effects of an internal heat deposition on a lithium jet as happens on the IFMIF target.

\subsection{Characterization techniques}

Any ambitious and comprehensive research requires an accurate knowledge of the materials under study and test. Therefore, a number of characterization techniques are the key element in the global scheme of TechnoFusión. These techniques include mechanical testing (creep, nanoindentation, fatigue, etc.), compositional analysis (secondary ion mass spectrometry or SIMS and atomprobe tomography or APT), and structural characterization (energy filtered-transmission electron microscopy or EF-TEM and X-ray diffraction or XRD), as well as a number of material processing techniques (double beam focus ion beam or FIB). Additional systems will be used to characterize physical properties (electrical, dielectric, optical, etc.). Some of the mentioned techniques will be implemented to test the materials either in-beam-while the radiation actually hits the testing material- and in situ, inside the lithium loop.

\subsection{Computer simulation}

To study the conditions that cannot be reached experimentally and to accelerate the development of novel systems for a future commercial FPP, TechnoFusión stimulates an ambitious programme of computer simulations, combining the existing experience in the fusion field with resources from the National Supercomputation Network [4]. Its goals include the implementation of the global simulation of a commercial fusion reactor, the interpretation of results, the validation of numerical tools, and the development of new tools. Another indispensable goal is the creation of a data acquisition system and the visualisation of results.

\subsection{Remote handling techniques}

The description of these facilities is the objective of the next chapter.

\section{Experimentation area for remote handling}

The principal objective is to create a facility where tele-robotic tasks for the maintenance and repair of nuclear fusion installations (ITER, DEMO, and IFMIF) can be developed and tested. Furthermore, it has to serve as a platform to promote the development of required technologies and as a base to undertake experiments and activities (dissemination, training, and know-how transfer). RH activities will be carried out in two facilities within the future TechnoFusión com- 


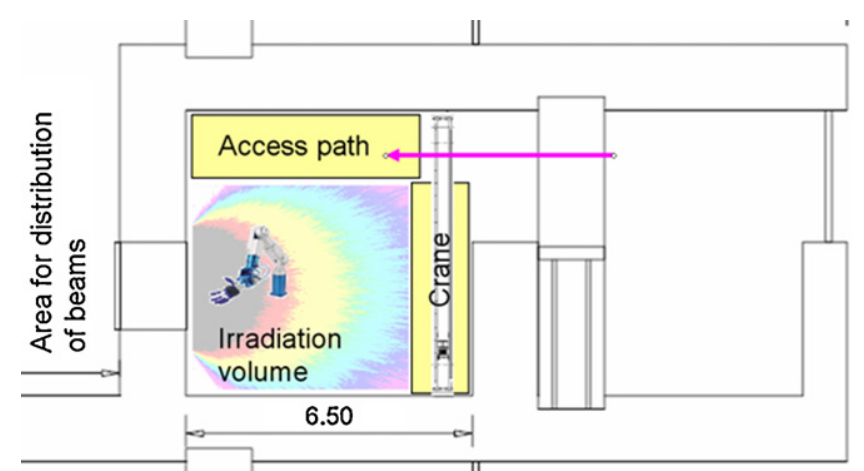

Fig. 1. Preliminary layout of the required irradiation room.

plex able to test large prototypes: a laboratory for irradiation test, and a remote handling design and development test facility.

\subsection{Laboratory for irradiation test}

The certification of RH equipment (i.e. robots, cranes and further equipments) will be performed testing and characterizing their individual components (i.e. encoders, cables, connectors, etc.) under irradiation. However, the reliability level of such independent tests will not assure the required reliability level of the whole manipulator under real working conditions. A large irradiating facility, able to irradiate the manipulators to a large extent while performing repetitive operations, is therefore required.

An effective irradiation volume of $5 \mathrm{~m} \times 5 \mathrm{~m} \times 4 \mathrm{~m}$ (Fig. 1) has been defined as a reasonable balance of several factors. This robot testing volume would result in higher performance and greater possibilities for irradiation. A $10 \mathrm{MeV}$ electron beam impacting on a tungsten layer has been selected to generate the required gamma radiation. Some parameters have been calculated in order to optimize the production of the gamma radiation from a practical, economical and technical point of view: the optimum gamma radiation dose, the maximum uniformity of the irradiation into the room and the maximum number of manipulators irradiated per year. An electron beam accelerator scanning on a $1500 \mathrm{~mm} \times 1500 \mathrm{~mm} \times 6 \mathrm{~mm}$ tungsten wall (beam incidence angle $53^{\circ}$ ) has been selected in the preliminary design. Beam sweeping

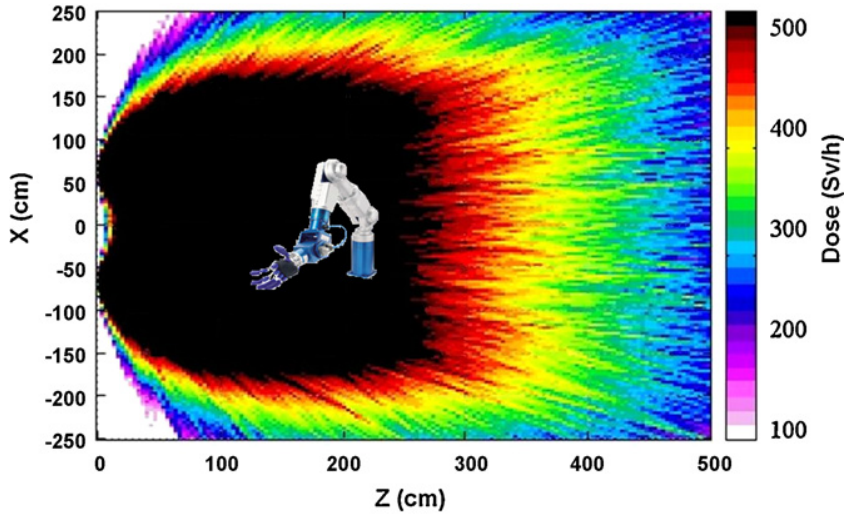

Fig. 2. Gamma dose simulation in a total effective volume of a $5 \mathrm{~m} \times 5 \mathrm{~m} \times 4 \mathrm{~m}$ irradiation room, considering a $10 \mathrm{MeV}$ electron beam scanning on a $1500 \mathrm{~mm} \times 1500 \mathrm{~mm} \times 6 \mathrm{~mm}$ tungsten wall.

should be concentrated on a relatively reduced area (about $4 \mathrm{~m}^{2}$ or less) from the wall of the gamma ray generator in order to obtain as high as 2000 or $3000 \mathrm{~Gy} / \mathrm{h}$ in a small volume inside the irradiation room. The gamma dose in the first row will be at least $500 \mathrm{~Gy} / \mathrm{h}$ (Fig. 2). The dose in the second row will at minimum be $100 \mathrm{~Gy} / \mathrm{h}$.

Additionally, the irradiation room will be equipped with auxiliary tools flexible enough to accommodate unknown future experiments. Cranes and stacking machines used to position small robots and robotic manipulator tools on structures such as shelves need to be considered. Standard wireless communication systems will need to be installed sufficiently flexible to client requirements. The room will permit the future installation of heating systems (testing conditions up to $70^{\circ} \mathrm{C}$ ).

\subsection{Remote handling design and development test facility}

It will be useful for the validation and improvement of RH procedures. The RH operations associated to mock up components will need to be physically demonstrated before they are accepted for installation. In addition, the development and improvement of the RH compatibility of the components to be remotely handled as well as the RH equipment and tools (e.g. master slave manipulator) for an enhanced RH maintenance is of critical importance. The

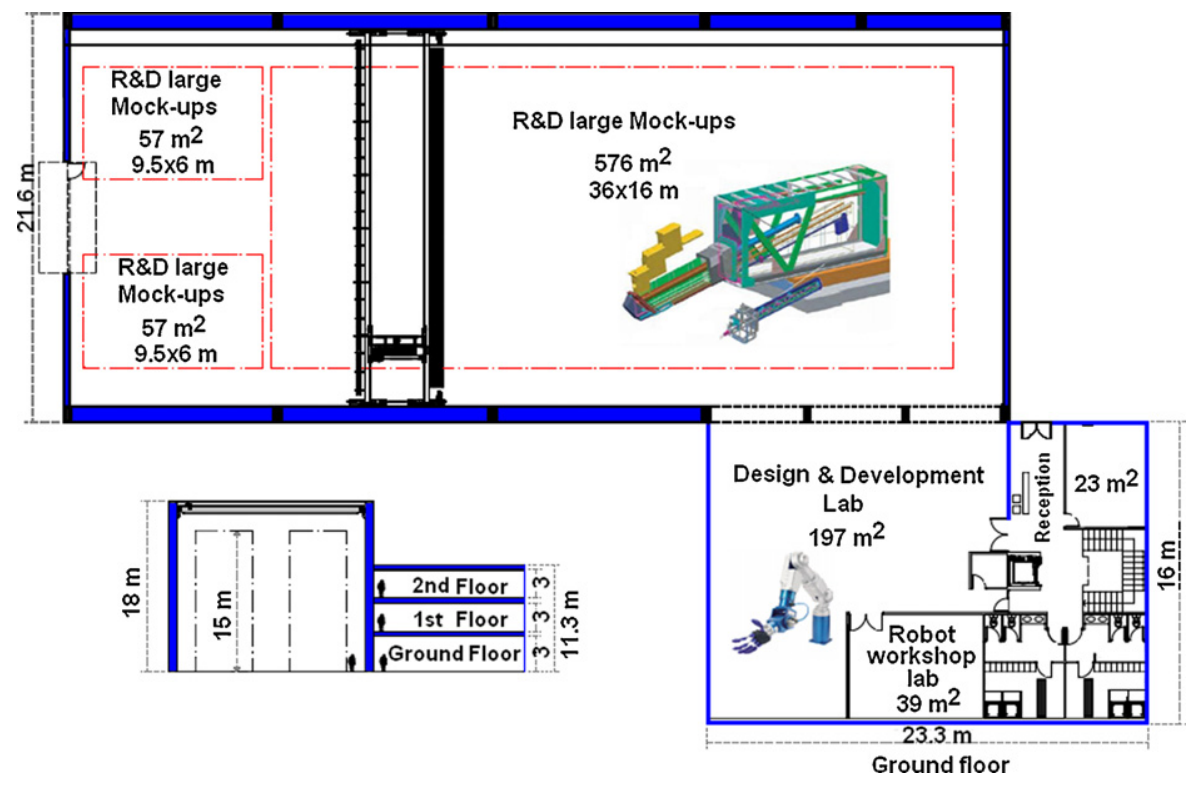

Fig. 3. Draft of the RH design and development test facility. 

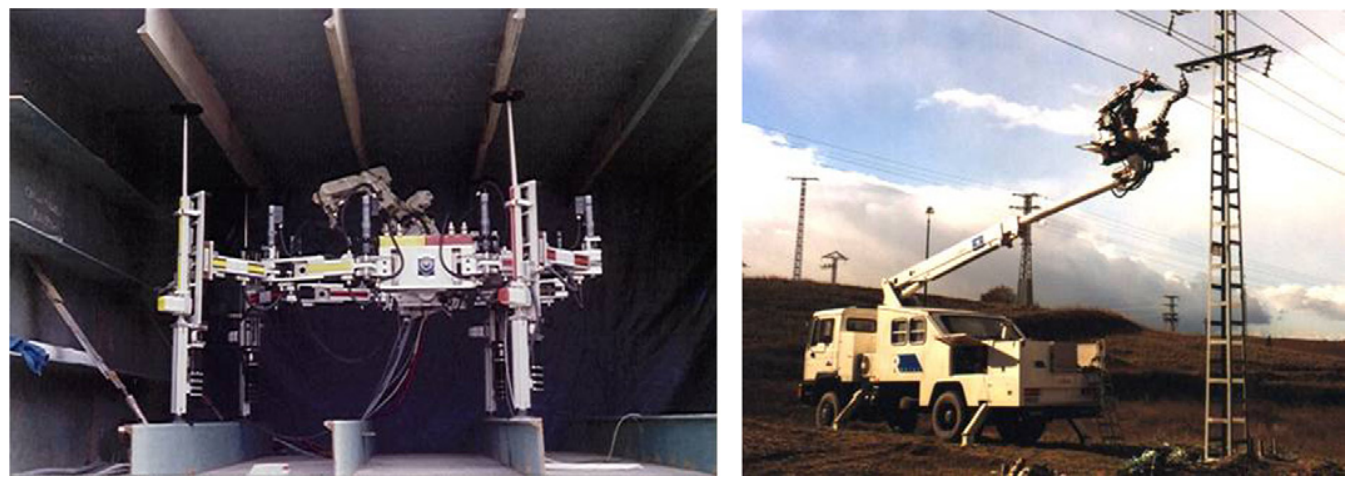

Fig. 4. ROWER designed for welding applications (left). ROBTET for live-line maintenance (right).

facility is meant to be used by different client projects needing to demonstrate the feasibility of the RH operations associated to their components.

The facility will consist of a large building of $50 \mathrm{~m} \times 21.6 \mathrm{~m}$ and $18 \mathrm{~m}$ high that will contain the mock-up components to be remotely manipulated and the $\mathrm{RH}$ equipment (Fig. 3 ). This building will also have a $400 \mathrm{~m}^{2}$ area for the development and improvement for RH technologies. Finally, there will be a control room from which all RH equipment in the facility will be manipulated.

One of the design requirements of the facility is the possibility to change quickly from one experimental set-up (mock-ups, supporting structures, specific RH equipment) to another without making significant changes in the building (floors, walls).

The facility will have some $\mathrm{RH}$ equipment permanently installed, likely to be used by more than one test client. Specific $\mathrm{RH}$ equipment of each test client project will need to be installed as needed. The permanent equipment will consist in: a crane of about 40 tons capable of vertical axis rotation of the load without swaying on the horizontal axis, a light force-reflecting commercial servo-manipulator, a power servo-manipulator, a RH vision system, a control system and human-machine interface (HMI) and finally a movable porch equipped with a telescopic mast so as to support and locate one of the servo-manipulators. The equipment accommodation area is a large space with a $50 \mathrm{~m} \times 20 \mathrm{~m}$ surface and $18 \mathrm{~m}$ high. This area is used for mock-ups to be remotely manipulated. Main equipments considered for this space are: a crane of 40 tons with 6 degree of freedom, two hydraulic powered telemanipulators placed on a mobile platform, and some devices for the human interface such as stereoscopic cameras.

Three laboratories are also included in this building; the whole space recommended will be close to $1000 \mathrm{~m}^{2}$.

\subsubsection{Laboratory of tele-operation and control}

Laboratory of tele-operation and control, where definition and testing of RH procedures will be held. It includes the 'Control Room' for the tasks concerning mock-ups. Works will be carried out mainly with the equipments in the accommodation area but it is also considered to remote control other devices located in different centres.

The lab will concentrate mainly in bilateral tele-robotic control system, which plays an important role in tele-operation since it is the way to link master and slave devices to efficiently manipulate remote objects. The master has a twofold function: to follow operator hand movements and reflect the interaction forces from the slave side. In such a manner, the operator knows the consequences of his/her actions, which significantly facilitates tele-manipulation tasks. The slave robot is in charge of reproducing the operator hand movements according to the master commands and measuring contact forces produced upon manipulation. A large number of experiments highlight the higher performance of bilateral systems versus unilateral ones, which only transmit hand movements without force feedback information, or interfaces based on sensorial substitution, which inform about applied forces through different senses (touch, vision, etc.). Interest for these types of devices can be seen in Goertz early works [5].

\subsubsection{Laboratory of virtual reality}

Virtual reality (VR) and 3D virtual world technologies are focused on developments for the laboratory of tele-operation and control. From a reliability and safety point of view, many noncritical testing tasks will be validated by quasi-real experiments of maintenance operations. Final tuning of RH procedures in training facilities is essential to succeed during real operations. Therefore, a training platform based on VR to these people in charge of performing RH operations will be used. In order to obtain the most relevant experience, the VR platform development will imply the study of different research fields: (1) simulation of the action-reaction processes caused by the robotic actuators; (2) simulation of the real physical forces in order to create the feeling of being in the real world; (3) interaction with virtual objects, and (4) providing an accurate feedback by means of advanced user interfaces. Software tools like Marilou Robotics Studio ${ }^{\circledR} 2008$ [6] have been studied for this purpose. Nowadays, it is possible to use VR to improve real-time viewing systems required for $\mathrm{RH}$ operations [7].

Real-time camera views still remain as essential in the RH strategy in order to cope with unpredictable situations like machine damage or unexpected objects into the remote working site. However, the possibility of integrating sensor data (providing viewing systems, laser telemeters, force and position sensors) into a 3D virtual world is being considered to improve the performance of the VR system under development. The system is expected to assist monitoring under limited viewing information.

\subsubsection{Laboratory of robot design and development}

The maintenance of fusion facilities has to be done under special conditions: remote operation, irradiation environment, heavy load and big dimension component manipulation. However, many of the specific tasks to be accomplished have a direct relationship to standard industrial operations: inspection and measuring, welding and cutting, assembly and disassembly, fixing and removing. ... Therefore, relevant advances have to be taken to design industriallike robot systems or robotic structures that support adapted tools to work under such new and hard conditions.

Some topics to be implemented in TechnoFusión RH area are the following: control of water-hydraulic and electric articulations in high precision operations; force control of operations in 
robots mounted on non-rigid platforms; or component design constraints imposed by tele-operated robotic operations as in industrial workplaces. It is foreseen the need of designing different robotic structures for special purposes.

Previous experience with this kind of activities is shown in Fig. 4, which shows ROWER [8] and ROBTET [9] prototypes. ROWER, an example of a specific solution from industrial operations into nonindustrial environments, is a walking robot for shipyard work that transports a welding robot in the double hull of a ship. ROBTET is a tele-operated system for live-line maintenance. The operator controls the tele-manipulator from the cabin located on the track.

\section{Conclusions}

The conditions inside a fusion reactor are incompatible with a manual repair or replacement of parts. Therefore, new robotic techniques, compatible with such hostile conditions, need to be developed while existing techniques need certification in order to be applied at installations such as DEMO or IFMIF. TechnoFusión facility will contribute to this knowledge with:

(i) A laboratory for irradiation tests (coupled to an electron accelerator) to carry out validation, certification and characterization of $\mathrm{RH}$ tools and prototypes in an uniform ionizing field equivalent to ITER-DEMO trying to simulate the fusion reactor environment. (ii) A large installation for prototype manipulation. The size of components and prototypes to be manipulated and the complications associated with their spatial location will require the development of new remote handling techniques.

\section{Acknowledgments}

This work was funded by the Spanish Science and Innovation Ministry (MICINN) under grant ICTS-2008-9 and under the National Spanish Plan Radiafus II (CICYD Project ENE2008-06403-C06-01).

\section{References}

[1] K. Lackner, R. Andreani, D. Campbell, M. Gasparotto, D. Maisonnier, M.A. Pick, Journal of Nuclear Materials 307-311 (2002) 10-20.

[2] K. Ehrlich, E.E. Bloom, T. Kondo, Journal of Nuclear Materials 283 (2000) 79-88.

[3] D. Maisonnier, I. Cook, S. Pierre, B. Lorenzo, D.P. Luigi, G. Luciano, et al., Fusion Engineering and Design 81 (2006) 1123-1130.

[4] http://www.bsc.es/index.php, September 2009.

[5] R. Goertz, Manipulator systems development at ANL, in: Proc. of the 12th Conf on Remote Systems Technology, 1964, ANS: 117-136.

[6] http://files.marilou-roboticsstudio.com/MarilouInstall 20071021 L17 D3DX.zip.

[7] S. Sanders, A.C. Rolfe, The use of virtual reality for preparation and implementation of JET remote handling operations, in: 22nd Symposium on Fusion Technology, Helsinki, Finland, 2002.

[8] P. Gonzalez de Santos, M. Armada, M.A. Jimenez, Ship building with ROWER, IEEE Robotics and Automation Magazine 7 (4) (2000) 35-43.

[9] R. Aracil, M. Ferre, M. Hernando, E. Pinto, J.M. Sebastian, Telerobotic system for live-power line maintenance:ROBTET, Control Engineering Practice 10 (2002) 1271-1281. 